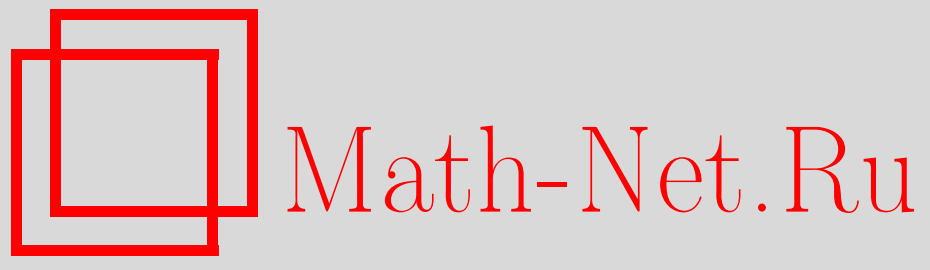

В. А. Ватутин, А. М. Зубков, Памяти Бориса Александровича Севастьянова, Теория вероятн. и ее примен., 2015, том 60, выпуск 1, 151-162

DOI: https://doi.org/10.4213/tvp4610

Использование Общероссийского математического портала Math-Net.Ru подразумевает, что вы прочитали и согласны с пользовательским соглашением http: //www . mathnet.ru/rus/agreement

Параметры загрузки:

IP : 54.198 .64 .247

26 апреля 2023 г., 15:55:36

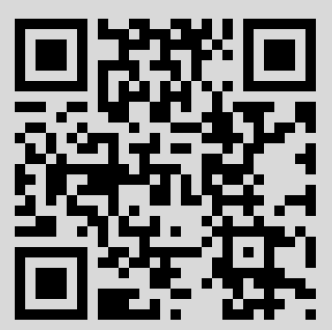


В качестве признания его вклада в алгебру и теорию вероятностей и обучение студентов в СССР и США он был удостоен премии Leroy P. Steele Prize for Lifetime Achievement от американского математического общества в 1993 году.

Светлая память о Евгении Борисовиче Дынкине сохранится в сердцах его друзей, коллег и учеников.

\section{Памяти Бориса Александровича Севастьянова}

(29.09.1923-30.08.2013)

30 августа 2013 на 90-м году жизни скончался член-корреспондент Российской академии наук Борис Александрович Севастьянов.

Б. А. Севастьянов родился в Москве 29 сентября 1923 года. После окончания школы в 1941 году он поступил на механико-математический факультет Московского университета. В связи с Великой Отечественной войной обучение на мех-мате завершилось лишь в 1948 году.

Будучи студентом, Борис Александрович посещал различные спецкурсы и спецсеминары, пытаясь найти тот раздел математики, который бы больше всего ему подходил. Сначала его привлекла красота комбинаторной топологии, и на третьем курсе Севастьянов сделал реферативный доклад о сферах с ручками на спецсеминаре Льва Семеновича Понтрягина. Однако окончательный выбор кафедры он сделал осенью 1946 года, заинтересовавшись тематикой традиционного семинара по теории вероятностей, который вел А.Н. Колмогоров. Среди участников семинара были как ученики Колмогорова, так и сотрудники кафедры теории вероятностей, в число которых входили Е. Б. Дынкин, Н. А. Дмитриев, А. С. Монин, А. М. Яглом. Именно на этом семинаре и были заложены основы одного из важных направлений теории вероятностей, носящего в настоящее время название «Теория ветвящихся процессов». Даже сам термин «ветвящийся процесс» впервые появился в работе А. Н. Колмогорова и Н. А. Дмитриева «Ветвящиеся случайные процессы», опубликованной в 1947 году (Доклады АН СССР, 56 (1), стр. 7-10).

На одном из заседаний семинара Колмогоров рассказал о задаче вырождения фамилий и ее интерпретации в терминах размножения частиц и, среди прочего, поставил задачу нахождения условий вырождения семейств, состоящих из двух типов частиц. После того, как Борис Александрович решил эту задачу, Колмогоров предложил рассмотреть проблему вырождения семейств, состоящих из нескольких типов частиц, которую Борис Александрович также решил. В результате на свет появилась первая печатная работа Б. А. Севастьянова «Вычисление финальных вероятностей для ветвящихся случайных процессов» [1], в соавторстве с академиком А.Н. Колмогоровым. Вот как описывал это событие сам Борис Александрович в своих воспоминаниях [105]: 
«...После летних каникул, в сентябре 1947 г., Андрей Николаевич показал мне только что вышедшую в «Докладах Академии наук», статью «Вычисление финальных вероятностей для ветвящихся случайных процессов», где я значился его соавтором. Я сначала даже не понял, как это я оказался соавтором работы Андрея Николаевича. Как вообе об этом могла идти речь! Вся работа была написана Андреем Николаевичем, а в середине статьи один абзац начинался словами: «Младшим из авторов настоящей заметки доказана следующая теорема...». Далее шла формулировка теоремы, и сообщалось, что «...доказательство теоремы будет опубликовано в другом месте». Формулировка этой теоремы несколько отличалась от той, что я предложил тогда на семинаре, однако мой метод доказательства был применим и к этой новой теореме. В этой работе Колмогоров ввел понятие финальных частиц, представляющих собой конечный продукт реакций, моделью которых являются ветвящиеся процессы. Кроме того, Андреем Николаевичем в этой статье была намечена некоторая программа развития одного из направлений теории ветвящихся процессов. Впоследствии эта программа была мною выполнена и составила содержание одной из глав моей кандидатской диссертации. ..».

С 1948 по 1951 годы Борис Александрович проходил обучение в аспирантуре в НИИ математики МГУ под руководством академика Андрея Николаевича Колмогорова и стал одним из его первых послевоенных учеников (см. воспоминания [77], [91], [100], [105]). Осенью 1948 года Борис Александрович стал младшим научным сотрудником Математического института им. В.А. Стеклова АН СССР; впоследствии он был старшим научным сотрудником, заведующим лабораторией, заведующим отделом Математического института.

В 1952 году Борис Александрович защитил кандидатскую диссертацию под названием «Теория ветвящихся процессов», в которой дано систематическое изложение теории ветвящихся процессов с одним или несколькими типами частиц и непрерывным или дискретным временем. Им были получены функциональные уравнения для производящих функций распределений числа потомков частиц в неоднородных ветвящихся процессах, выведены дифференциальные уравнения в частных производных, которым удовлетворяют указанные производящие функции в случае процессов с непрерывным временем, получены уравнения для факториальных моментов числа частиц в процессах с одним типом частиц, указан способ нахождения вероятности вырождения процессов с непрерывным и с дискретным временем, получены асимптотические представления для вероятностей невырождения докритических и критических процессов с непрерывным временем, доказаны условные предельные теоремы о распределении числа частиц в докритических и критических процессах при условии невырождения к далекому моменту времени, при- 
ведена классификация ветвящихся процессов с несколькими типами частиц, рассмотрены задачи вычисления вероятностей вырождения таких процессов и приведен критерий вырождения ветвящихся процессов с несколькими типами частиц. По сути дела в этой работе были получены фундаментальные результаты многих направлений зарождавшейся теории ветвящихся процессов.

В 1957 году в работе [13] Борис Александрович ввел и проанализировал следующую модель ветвящихся процессов с иммиграцией. В этой модели каждая из существующих в данный момент частиц независимо от своего происхождения и возраста и судьбы других частиц с вероятностью $\delta_{k 1}+p_{k} \Delta t+o(\Delta t)$ превращается за промежуток времени $\Delta t \rightarrow 0$ в $k$ частиц, и, кроме того, независимо от наличия какого-либо числа частиц в процессе, в популяцию с вероятностью $\delta_{k 0}+q_{k} \Delta t+o(\Delta t)$ за промежуток времени $\Delta t \rightarrow 0$ иммигрируют $k$ частиц. Пусть

$$
f(s)=\sum_{k=0}^{\infty} p_{k} s^{k}, \quad g(s)=\sum_{k=0}^{\infty} q_{k} s^{k} .
$$

Предполагая, что $f^{(3)}(1)<\infty$ и $g^{(2)}(1)<\infty$, Борис Александрович вывел точные формулы для математического ожидания и дисперсии числа частиц, существующих в процессе в момент $t$, а также доказал предельные теоремы о распределения числа частиц при $t \rightarrow \infty$.

В статьях [11] и [15] Борис Александрович изучал так называемые переходные явления для однородных марковских ветвящихся процессов с одним типом частиц и непрерывным временем. Известно, что свойства таких процессов во многом определяются параметром $a=f^{\prime}(1)$. Предполагая, что $a \rightarrow 0$, a $t \rightarrow \infty$ согласованным образом, Борис Александрович нашел асимптотическое представление для величины $Q(t, a)$ - вероятности невырождения процесса к моменту $t$, а также доказал предельную теорему о распределении числа частиц в такой модели при условии невырождения процесса к моменту $t$ и предположении, что первоначальное число частиц $n$ в процессе и момент наблюдения $t$ стремятся к бесконечности так, что $n Q(t, a) \rightarrow \gamma \in(0, \infty)$.

$\mathrm{K}$ числу важнейших работ Севастьянова следует отнести статьи [14] и [19], в которых рассматриваются ветвящиеся процессы с диффузией. В этой модели частица типа $T_{i}, i=1, \ldots, m$, диффундирует с коэффициентом диффузии $D_{i}>0$ в $r$-мерной области $G$ с поглощающей границей Г. При помощи процесса блуждания строится ветвящийся процесс с $m$ типами частиц и перемещением частиц в пространстве. В этом ветвящемся процессе время жизни $\tau_{x i}$ частицы типа $T_{i}$, начинающей свое блуждание из точки $x \in G, \operatorname{paвно} \min \left(\tau_{x i}^{*}, \tau_{i}\right)$, где случайные величины $\tau_{x i}^{*}$ и $\tau_{i}$ независимы, причем $\tau_{i}$ имеет экспоненциальное распределение с параметром $c_{i}$, а $\tau_{x i}^{*}$ - время блужданий частицы из точки $x$ до момента поглощения на границе. Если $\tau_{x i}=\tau_{x i}^{*}$, то частица погибает на 
границе области без потомства, если же $\tau_{x i}=\tau_{i}$, то в конце жизни частица типа $T_{i}$ производит потомков различных типов в соответствии с производящей функцией $F_{i}\left(s_{1}, \ldots, s_{m}\right)$. В частности, с вероятностью $F_{i}(0, \ldots, 0)$ частица не оставляет потомства. Для такой модели были выведены дифференциальные уравнения для производящих функций числа частиц в момент $t$, получены асимптотические представления для факториальных моментов числа частиц, указаны необходимые и достаточные условия вырождения такого процесса с вероятностью единица, найдены асимптотические представления для распределения вероятностей совокупного числа частиц некоторых типов, появившихся в процессе за время его существования.

В 1964 году Борис Александрович предложил в работе [28] новую модель ветвящихся процессов с превращениями, зависящими от возраста частиц. Эта модель ветвящихся процессов в настоящее время носит название «процессы Севастьянова».

Процессы Севастьянова с $n$ типами частиц $T_{1}, \ldots, T_{n}$ можно неформально описать следующим образом. Каждая из частиц типа $T_{j}, j=$ $1,2, \ldots, n$, имеет случайную продолжительность жизни с функцией распределения $G_{j}(t), t \geq 0$. В конце жизни частица типа $T_{j}$ превращается в какую-нибудь совокупность частиц типов $T_{1}, \ldots, T_{n}$, причем если превращение произошло, когда частица достигла возраста $u$, то распределение $n$-мерного вектора числа ее потомков различных типов зависит от $u$. Борис Александрович исследовал в [28], [31] и [33] условия невырождения таких процессов и для критических процессов с одним типом частиц нашел асимптотику вероятности невырождения к далекому моменту $t$ и доказал условную предельную теорему о распределении числа частиц в таких процессах при условии их невырождения к моменту $t \rightarrow \infty$. В работе [49], написанной совместно с В.П. Чистяковым, для ветвящихся процессов с несколькими типами частиц и превращениями, зависящими от возраста частиц, авторы изучили асимптотическое поведение моментов числа частиц различных типов для надкритических, критических и докритических процессов. В этой работе были использованы теоремы Б. А. Севастьянова, относящиеся к обобщению теорем восстановления на многомерный случай [41], [45].

Частным случаем процессов Севастьянова являются процессы Беллмана-Харриса, в которых распределение числа потомков частиц не зависит от длительности жизни частиц-родителей. Процесс БеллманаХарриса $Z(t), t \geq 0$, с одним типом частиц называется регулярным, если $\mathbf{P}(Z(t)<\infty)=1$ для любого $t>0$, и нерегулярным в противном случае. В работах [35] и [47] Б. А. Севастьянов описал достаточные (а в некоторых случаях необходимые и достаточные) условия нерегулярности процессов Беллмана-Харриса. 
Борис Александрович является основателем отечественной школы ветвящихся процессов, его результаты в этой области оказали существенное влияние на развитие теории ветвящихся процессов во всем мире. Результаты по теории ветвящихся процессов, полученные Севастьяновым, нашли свое отражение в монографии Бориса Александровича «Ветвящиеся процессы» (1971 г., [48]), которая была переведена на немецкий и японский языки [57], [59], [61]. Лишь сравнительно недавно дальнейшее развитие теории ветвящихся процессов, порожденное ее новыми приложениями в химии и биологии, привело к возникновению существенно новых направлений: ветвящихся случайных блужданий, мерозначных ветвящихся процессов, ветвящихся процессов со случайной средой. Исследования по теории ветвящихся процессов, начатые Борисом Александровичем, были продолжены в работах его сотрудников и учеников: В. П. Чистякова, В.Ф. Колчина, А. М. Зубкова, В. А. Ватутина, Е. Е. Дьяконовой, О. В. Вьюгина, С. А. Гришечкина, Г. Д. Макарова, А. В. Калинкина, А. Л. Якымива, а также в работах ряда математиков, работавших в различных республиках Советского Союза и за рубежом: О. П. Виноградова, В. М. Золотарева, А. В. Нагаева, С. В. Нагаева, Б. П. Харламова, И. С. Бадалбаева, В. М. Шуренкова, Р. Ибрагимова, И. Рахимова, Ш. К. Форманова, А. К. Полина, С. М. Сагитова, В. А. Топчия, Н. М. Янева (Болгария), П. Ягерса (Швеция) и др.

Научные интересы Бориса Александровича не ограничивались теорией ветвящихся процессов. И в других областях теории вероятностей он получил результаты, которые стали классическими.

В [9] Борис Александрович доказал общую теорему об условиях эргодичности марковского процесса и с ее помощью нашел стационарное распределение числа занятых линий в системе массового обслуживания типа $M|G| n$ с потерями: если в систему, состоящую из $n$ параллельно работающих устройств, поступает пуассоновский поток заявок с интенсивностью $\lambda$, а длительность обслуживания каждой заявки имеет произвольную функцию распределения $G(x)$ с конечным математическим ожиданием $\mu$, то плотность $p_{k}\left(x_{1}, \ldots, x_{k}\right)$ вероятности того, что в стационарном режиме в системе находится $k$ заявок, причем эти $k$ заявок уже обслуживаются $x_{1}, \ldots, x_{k}$ единиц времени соответственно, вычисляется по формуле

$$
\begin{gathered}
p_{k}\left(x_{1}, \ldots, x_{k}\right)=\frac{\lambda^{k}}{k !} p_{0} \prod_{i=1}^{k}\left(1-G\left(x_{i}\right)\right), \quad k=1,2, \ldots, n, \\
p_{0}=\left(\sum_{i=0}^{n} \frac{(\mu \lambda)^{i}}{i !}\right)^{-1} .
\end{gathered}
$$

Следствием этих формул явилась первая теорема инвариантности в теории массового обслуживания: вероятности $p_{k}$ того, что в стационар- 
ном режиме в системе находится $k$ заявок, вычисляются по формулам Эрланга

$$
p_{k}=\frac{(\lambda \mu)^{k} / k !}{\sum_{j=0}^{n}(\lambda \mu)^{j} / j !}, \quad k=0,1, \ldots, n
$$

при любом распределении времени обслуживания заявки с математическим ожиданием $\mu$. До этого формулы Эрланга были доказаны только в случае показательного распределения времени обслуживания.

Начиная с 60-х годов Б. А. Севастьянов вместе с группой математиков (В. Ф. Колчиным, В. П. Чистяковым, Ю. И. Медведевым, Ю. И. Ивченко и др.) разрабатывал теорию случайных размещений частиц по ячейкам (фактически - методы решения новых задач для полиномиальных распределений). До этого для частот $\eta_{1}(N), \eta_{2}(N), \ldots, \eta_{n}(N)$ исходов в $N$ испытаниях полиномиальной схемы с вероятностями исходов $p_{1}, \ldots, p_{n}$ были известны в основном теоремы об их асимптотической нормальности и о свойствах построенной по ним статистике Пирсона (при $N \rightarrow \infty, n=$ const), а также разрозненные результаты типа задач о днях рождения или собирания купонов в случае $p_{1}=\cdots=p_{n}$. Практические потребности построения статистических критериев на основе статистик, инвариантных относительно любых перенумераций исходов, в случаях, когда число испытаний $N$ и число исходов $n$ одновременно стремятся к бесконечности, привели к необходимости постановки и решения ряда новых задач, относящихся к распределениям случайных величин $\mu_{r}(n, N)=\sum_{k=1}^{n} \mathbb{I}\left\{\eta_{k}(N)=r\right\}$, т.е. чисел исходов, появившихся в $N$ испытаниях заданное число $r$ раз. В работах, выполненных Борисом Александровичем и его сотрудниками, был получен целый спектр теорем о сходимости распределений отдельных величин $\mu_{r}(n, N)$ и их конечных наборов к пуассоновским и нормальным законам при различных предположениях о соотношениях между $n$ и $N$ при $n, N \rightarrow \infty$ и об изменении набора вероятностей $p_{1}, \ldots, p_{n}$, а также теорем о сходимости последовательностей $\mu_{r}(n, N)$ к пуассоновским и гауссовским процессам при $n, N \rightarrow \infty$. Немного позже близкими вопросами стали заниматься и зарубежные математики.

Впоследствии это направление, казавшееся вначале довольно частным, стало существенной частью современной теории вероятностей. Опубликованная в 1976 году монография Бориса Александровича «Случайные размещения» [60], написанная в соавторстве с В.Ф.Колчиным и В. П. Чистяковым, вскоре была переведена в США; она дополняла более элементарную книгу Johnson N.L., Kotz S. «Urn Models and Their Applications» (J.Wiley \& Sons, 1977). Язык теории случайных размещений стал теперь общепринятым при постановке и решении задач в различных областях практического применения вероятностных и статистических методов. 
Одним из важных «побочных» результатов исследований по случайным размещениям частиц стала методика доказательства теорем о сходимости распределений сумм зависимых индикаторов к распределению Пуассона методом моментов, опубликованная Б. А. Севастьяновым в виде теоремы в 1972 году [50]. Впоследствии эта теорема многократно использовалась отечественными математиками при доказательстве самых разных теорем теории вероятностей и способствовала развитию отечественной вероятностно-комбинаторной школы. Позднее зарубежные математики Чен, Барбур, Янсон и др. разработали другой способ (метод Стейна-Чена) доказательства сходимости распределений сумм зависимых индикаторов к распределениям пуассоновского типа, позволяющий также оценивать скорость сходимости. Применение каждого из этих методов к конкретным задачам, как правило, связано с получением нетривиальных оценок.

Развитие различных областей математики и других наук во многом определяется практическими потребностями государств, промышленности, финансовых структур и т.п. С 50-х годов Б. А. Севастьянов (как и другие отечественные и зарубежные ученые на протяжении нескольких последних веков) привлекался к исследованиям в области криптографии, которая является источником разнообразных и трудных математических задач. С этими приложениями математики связан ряд публикаций Бориса Александровича по случайным отображениям конечных множеств [89], [98], [101], [103], по вероятностным вопросам теории булевых функций [86], [96], по теории случайных автоматов [83], [84], [97]. Например, в [83] и [97] рассматривалась статистическая задача проверки сложной гипотезы о наличии связи между двоичными последовательностями $\left\{x_{t}\right\}$ и $\left\{y_{t}\right\}$ вида $y_{t}=f_{k}\left(x_{t}, x_{t+1}, \ldots, x_{t+n-1}\right)$ (где $f_{k}$ - неизвестная функция из известного класса функций, число элементов которого очень велико) по статистикам $\left(y_{t}, x_{t}+x_{t+1}+\cdots+x_{t+n-1}\right)$ или $\left(y_{t}, y_{t+1}, x_{t}+x_{t+1}+\cdots+x_{t+n-1}+x_{t+n}\right)$, в которых величины $x_{k}$ рассматриваются как целые числа.

Интересные результаты были получены Борисом Александровичем по распределениям перманентов матриц над конечными полями [80], [87], [93], [108].

Наряду с интенсивной научной работой Борис Александрович вел активную преподавательскую деятельность. В 1952 году он стал (по совместительству) доцентом кафедры теории вероятностей механикоматематического факультета МГУ, в 1968 году — профессором. Учебники Б. А. Севастьянова «Курс теории вероятностей и математической статистики» [69], [102], «Теория вероятностей» (совместно с В. К. Захаровым, О.В. Сармановым) [71], [73], [76], а также «Сборник задач по теории вероятностей» (совместно с А. М. Зубковым, В.П. Чистяковым) $[66],[72],[75],[79],[107]$ используются в различных университетах на- 
шей страны и за рубежом. Борис Александрович был членом советов по защите дисссертаций в Математическом институте и в Московском государственном университете им. М. В. Ломоносова, работал в Экспертном совете ВАК, много раз был официальным оппонентом и не боялся давать отрицательные отзывы о диссертациях низкого качества.

Борис Александрович входил в редакционные коллегии журналов «Теория вероятностей и ее применения», «Дискретная математика», «Труды по дискретной математике» и «Математические вопросы криптографии» с их основания.

В 1984 году Борис Александрович был избран членом-корреспондентом АН СССР, а в 1991 году он принял активное участие в организации Академии криптографии Российской Федерации и стал ее действительным членом.

Заслуги Бориса Александровича Севастьянова были отмечены высокими государственными наградами: орденом Знак Почета (1976 г.), орденом Трудового Красного Знамени (1982г.), Государственной премией (1990 г.). В 1999 году Борис Александрович был удостоен степени почетного доктора («Honoris causa») Чалмерского технологического университета (Гетеборг, Швеция).

Разнообразие научных интересов Бориса Александровича, его интенсивная исследовательская деятельность (в списке публикаций содержится более 100 статей и монографий с 1947 по 2010 год), умение ставить математические задачи в привлекательной форме, а также такие человеческие качества, как прямота и бескомпромиссность, объединяли вокруг него других математиков и единомышленников. Он сформировал научный профиль Отдела дискретной математики Математического института им. В. А. Стеклова.

Светлая память о Борисе Александровиче Севастьянове, замечательном ученом, прекрасном преподавателе и просто порядочном человеке навсегда сохранится в сердцах всех тех, кто его знал.

\section{СПИСОК ЛИТЕРАТУРЫ}

1. Вычисление финальных вероятностей для ветвящихся случайных процессов. Докл. АН СССР, 1947, т. 56, № 8, с. 783-786 (совм. с А. Н. Колмогоровым).

2. Ветвящиеся случайные процессы. - Вестник МГУ, 1948, № 3, с. 13-34.

3. К теории ветвящихся случайных процессов. - Докл. АН СССР, 1948, т. 59, № 8, с. $1407-1410$.

4. Теория ветвящихся случайных процессов. - Успехи матем. наук, 1951, т. 6, в. 6 , с. $47-99$.

5. Ветвящиеся случайные процессы. - Диссертация на соискание ученой степени кандидата физ.-матем. наук. М.: МГУ, 1951, 79 с.

6. Математическая статистика и контроль промышленной продукции. - Природа, 1955, № 8, с. 28-34 (совм. с С. Х. Сираждиновым). 
7. Statistica mathematica si controlul productiei industriale. - Anal. Romino-Soviet. Matem.-Fiz., 1956, № 3, c. 33-42 (with S. H. Sirajdinov).

8. Теория вероятностей. - Большая советская энциклопедия. 2-е изд., т. 42, М.: Сов. энциклопедия, 1956, с. 230-236 (совм. с Ю. В. Прохоровым).

9. Эргодическая теорема для марковских процессов и ее приложение к телефонным системам с отказами. - Теория вероятн. и ее примен., 1957, т. 2, в. 1, с. 106-116.

10. Формулы Эрланга в телефонии при произвольном законе распределения длительности разговора. - Теория вероятн. и ее примен., 1957, т. 2, в. 1, с. 135.

11. Переходные явления в ветвящихся случайных процессах. - Теория вероятн. и ее примен., 1957, т. 2, в. 1, с. 136-138.

12. Финальные вероятности ветвящихся случайных процессов. - Теория вероятн. и ее примен., 1957, т. 2, в. 1, с. 140-141.

13. Предельные теоремы для ветвящихся случайных процессов специального вида. - Теория вероятн. и ее примен., 1957, т. 2, в. 3, с. 339-348.

14. Ветвящиеся случайные процессы для частиц, диффундирующих в ограниченной области с поглощающими границами. - Теория вероятн. и ее примен., 1958, т. 3, в. 2, с. 121-136.

15. Переходные явления в ветвящихся случайных процессах. - Теория вероятн. и ее примен., 1959, т. 4, в. 2, с. 121-135.

16. Формулы Эрланга в телефонии при произвольном законе распределения длительности разговора. - В кн.: Труды Третьего всесоюзн. матем. съезда (Москва, июнь-июль 1956), т. 4. М.: Изд-во АН СССР, 1959, с. 68-70.

17. Ветвящиеся случайные процессы. - Физический энциклопедический словарь, т. 1. М.: Сов. энциклопедия, 1960, с. 253-254.

18. Доверительные пределы для средней вероятности в схеме Пуассона. - Теория вероятн. и ее примен., 1961, т. 6, в. 2, с. 242-244.

19. Условия вырождения ветвящихся процессов с диффузией. - Теория вероятн. и ее примен., 1961, т. 6 , в. 3, с. $276-286$.

20. Класс предельных законов распределения квадратичных форм от нормальных случайных величин. - Теория вероятн. и ее примен., 1961, т. 6, в. 3, с. 368-372.

21. Задача о влиянии емкости бункеров на среднее время простоя автоматической линии станков. - Теория вероятн. и ее примен., 1962, т. 7, в. 4, с. 438-447.

22. Предельные теоремы для ветвящихся случайных процессов с диффузией. - In: Intern. Congress Mathemat. (Stockholm, 1962), Abstr. Short Commun., p. 168.

23. Предельные теоремы для ветвящихся процессов с диффузией. - В сб.: Труды VI Всесоюзн. совещ. по теории вероятн. и матем. статистике (ВильнюсПаланга, 5-14 сентября 1960 г.). Вильнюс: Изд-во полит. и научн. литературы, 1962 , с. $157-158$.

24. О многомерных концентрациях. - Теория вероятн. и ее примен., 1963, т. 8, в. 1, c. $124-125$.

25. Об одном неравенстве для двумерных законов распределения. - В сб.: Предельные теоремы теории вероятностей. Ташкент, АН УзССР, 1963, с. 118-122.

26. Об общем определении ветвящихся случайных процессов. - В сб.: Зимняя школа по теории вероятн. и матем. статистике (Ужгород, 1964). Киев: Издво АН УССР, 1964, с. 217-220.

27. Асимптотическая нормальность в классической задаче о дробинках. - Теория вероятн. и ее примен., 1964, т. 9, в. 2, с. 223-237; исправл.: 1964, т. 9, вып. 3, с. 568 (совм. с В. П. Чистяковым).

28. Ветвящиеся процессы с превращениями, зависящими от возраста частиц. Теория вероятн. и ее примен., 1964, т. 9, в. 4, с. 577-594; исправл: 1966 , т. 11, в. 2, с. 363-364.

29. Исследование и оценка продуктивности зрения. - Механизмы кодирования зрительной информации. М.: Наука, 1966, с. 80-89 (совм. с А. И. Коганом).

30. Предельные теоремы в одной схеме размещения частиц по ячейкам. - Теория вероятн. и ее примен., 1966, т. 11, в. 4, с. 696-700.

31. Асимптотика критических ветвящихся процессов. - В кн.: Междунар. конгресс математиков в Москве. Тезисы кратких научных сообщений. М.: 1966, с. 51.

32. Сходимость к гауссовскому и пуассоновскому процессам распределения числа 
пустых ящиков в классической задаче о дробинках. - Теория вероятн. и ее примен., 1967, т. 12, в. 1, с. 144-154.

33. Асимптотика вероятности продолжения критического ветвящегося процесса. Теория вероятн. и ее примен., 1967, т. 12, в. 1, с. 179-183.

34. Использование логарифмической трансформации при разработке стандартов физического развития детей и подростков. - Вопросы антропологии, 1967, № 25, с. 8-24 (совм. с В. Г. Властовским).

35. О регулярности ветвящихся процессов. - Матем. заметки, 1967 , т. 1, № 1, с. 5362.

36. О предельном поведении максимума в полиномиальной схеме. - Матем. заметки, 1967, т. 1, № 3, с. 331-338 (совм. с И. И. Викторовой).

37. Размещение случайного числа частиц по ячейкам. - Матем. заметки, 1967, т. 1, № 5, с. 549-554 (совм. с Г. И. Ивченко, Ю. И. Медведевым).

38. Теория ветвящихся процессов. - В кн.: Теория вероятностей. Математическая статистика. Теоретическая кибернетика. М.: ВИНИТИ, 1968, с. 5-46.

39. Ветвящиеся процессы. - Дисс. на соискание ученой степени доктора физ.матем. наук. М.: Матем. ин-т им. В. А. Стеклова АН СССР, 1968, 218 с.

40. Предельные теоремы для ветвящихся процессов с превращениями, зависящими от возраста частиц. - Теория вероятн. и ее примен., 1968, т. 13, в. 2, с. 243-265.

41. Уравнения типа восстановления и моменты ветвящихся процессов. - Матем. заметки, 1968, т. 3, № 1, с. 3-14.

42. Математические ожидания функций от сумм случайного числа независимых слагаемых. - Матем. заметки, 1968, т. 3, № 4, с. 387-394.

43. Ветвящиеся процессы (Автореферат докторской диссертации). - Матем. заметки, 1968, т. 4, № 2, с. 239-251.

44. Последовательный критерий хи-квадрат. - Матем. сб., 1969, т. 79, № 3, с. 444460 (совм. с В. К. Захаровым и О. В. Сармановым).

45. Уравнения многомерного восстановления и моменты ветвящихся процессов. В кн.: Советско-японский симпозиум по теории вероятн. (Хабаровск, 1969), т. 2. Новосибирск, 1969, с. 253-283 (совм. с В. П. Чистяковым).

46. Критерий «пустых ящиков» и его обобщения. - Труды Ин-та прикл. матем. Тбилисского гос. ун-та, 1969, т. 2, с. 229-238.

47. Необходимое условие регулярности ветвящихся процессов. - Матем. заметки, 1970, т. 7, № 4, с. 389-396.

48. Ветвящиеся процессы. М.: Наука, 1971, 436 с.

49. Уравнения многомерного восстановления и моменты ветвящихся процессов. Теория вероятн. и ее примен., 1971, т. 16, в. 2, с. 201-217 (совм. с В. П. Чистяковым).

50. Случайные отображения и разбиения конечных множеств. - Теория вероятн. и ее примен., 1972, т. 17, в. 1, с. 129-142.

51. Применение системы массового обслуживания с очередью для расчета одной простой экономической модели производства и потребления. - В сб.: Всесоюзн. научно-техн. конф. «Применение теории вероятностей и математической статистики в народном хозяйстве». Кишинев, 1972, с. 215-216.

52. Предельный закон Пуассона в схеме сумм зависимых случайных величин. Теория вероятн. и ее примен., 1972, т. 17, в. 4, с. 733-738.

53. Некоторые комбинаторные тождества. - Матем. заметки, 1972, т. 11, № 1, с. 121-127 (совм. с О. В. Сармановым и В. Е. Таракановым).

54. Some generalizations of branching processes. - In: Second Japan-USSR Symposium on Probability Theory. V. 1. Kyoto, 1972, p. 61-68.

55. Регулируемые ветвящиеся процессы. - В сб.: Междунар. конф. по теории вероятн. и матем. статистике (Вильнюс, 1973). Тезисы докладов. Т. 2. Вильнюс, 1973, с. 223-224 (совм. с А. М. Зубковым).

56. Регулируемые ветвящиеся процессы. - Теория вероятн. и ее примен., 1974, т. 19, в. 1, с. 15-25 (совм. с А. М. Зубковым).

57. Verzweigungsprozesse. Akademie-Verlag, Berlin, 1974. xi+326 pp.

58. Теория восстановления. - В кн.: Итоги науки и техники. Сер. Теория вероятн., матем. статист., теорет. киберн. Т. 11. М.: ВИНИТИ, 1974, с. 99-128. 
59. Verzweigungsprozesse. R. Oldenbourg Verlag, Munich-Vienna, 1975. xi+326 pp.

60. Случайные размещения. М.: Наука, 1976, 224 с. (совм. с В.Ф. Колчиным и В. П. Чистяковым).

61. Ветвящиеся процессы (на японском языке). 1976, 374 с.

62. Вероятностей теория. - В кн.: Математическая энциклопедия. Т. 1. М.: Сов. энциклопедия, 1977, с. 655-666 (совм. с Ю. В. Прохоровым).

63. Ветвящийся процесс. - В кн.: Математическая энциклопедия. Т. 1. М.: Сов. энциклопедия, 1977 , с. 681-684.

64. Random Allocations. (Translated from Russian.) J. Winston \& Sons, Washington, 1978. xi+262 pp. (with V.F. Kolchin, V. P. Chistyakov).

65. Ограниченные снизу ветвящиеся процессы. - Докл. АН СССР, 1978, т. 238, № 4, с. 811-813.

66. Сборник задач по теории вероятностей. М.: Наука, 1980, 224 с. (совм. с В. П. Чистяковым, А. М. Зубковым).

67. Об одной схеме зависимых размещений. - Изв. АН УзССР, сер. физ.-матем. наук, 1981, № 2, с. 7-41.

68. Ветвящиеся процессы с взаимодействием частиц. - В кн.: Третья Междунар. Вильнюсская конф. по теории вероятн. и матем. статистике. Тезисы докладов, т. 2. Вильнюс, 1981, с. 139-140.

69. Курс теории вероятностей и математической статистики. - М.: Наука, 1982, $255 \mathrm{c}$.

70. Ветвящиеся случайные процессы с взаимодействием частиц. - Докл. АН СССР, 1982, т. 264, № 2, с. 306-308 (совм. с А. В. Калинкиным).

71. Теория вероятностей. М.: Наука, 1983, 160 с. (совм. с В.К. Захаровым и В. П. Чистяковым).

72. Problems in the Theory of Probability. (Translated from Russian.) Moscow: Mir, 1985, 236 p. (with V.P. Chistyakov, A. M. Zubkov).

73. Teoria de las probabilidades. (Translated from Russian.) Moscow: Mir, 1985, 152 p. (with V.K. Zaharov, V.P. Chistyakov).

74. Процесс хи-квадрат и его применение в статистике. - В сб.: Вероятностые распределения и математическая статистика. Ташкент: Фан, 1986, с. 188-198 (совм. с Г. И. Ивченко).

75. Valoszinuseg-elmeleti feladatok. (Translated from Russian.) Budapest: Tankonyvkiado, 1987, 354 p. (with V.P. Chistyakov, A. M. Zubkov).

76. Probability Theory for Engineers. (Translated from Russian.) New York: Optimization Software, 1987, 161 p. (with V. K. Zaharov, V.P. Chistyakov).

77. Исследования по ветвящимся процессам на кафедре теории вероятностей МГУ. - Теория вероятн. и ее примен., 1989, т. 34, в. 1, с. 231-236.

78. О высоте случайных деревьев. - 5-я междунар. Вильнюсская конф. по теории вероятн. и матем. статистике, Вильнюс, 26 июня-1 июля 1989 г. Тезисы докладов, т. 4 , с. 218-219.

79. Сборник задач по теории вероятностей. 2-е изд. М.: Наука, 1989, 320 с. (совм. с В. П. Чистяковым, А. М. Зубковым).

80. Распределение вероятностей перманента случайной булевой матрицы. - Дискретн. матем., 1990, т. 2, № 2, с. 138-144 (совм. с Л. А. Ляпковым).

81. Вероятностные модели. М.: Наука, 1992, 175 с.

82. Распределение высоты случайного дерева с помеченными ребрами. - Теория вероятн. и ее примен., 1993, т. 38, в. 2, с. 453-457.

83. Условное распределение выхода автоматов без памяти при заданных характеристиках входа. - Дискретн. матем., 1994, т. 6, № 1, с. 34-39.

84. О числе входных последовательностей, соответствующих выходной последовательности конечного автомата. - Обозр. прикл. и промышл. матем., 1994, т. 1, № 1, с. 96-107 (совм. с В. П. Чистяковым).

85. Вероятности вырождения ограниченных снизу ветвящихся процессов. - Теория вероятн. и ее примен., 1995, т. 40, в. 3, с. 556-564.

86. Вес $n$-мерного булева вектора и сложение по модулю $2^{n}$; обобщение на случай $m^{n}$. - Дискретн. матем., 1995, т. 7, № 3, с. 3-7.

87. Предельное распределение вероятностей перманента случайной матрицы в поле 
GF $(p)$. - Дискретн. матем., 1996, т. 8, № 2, с. 3-13 (совм. с Л. А. Ляпковым).

88. Вероятности вырождения ограниченных снизу докритических многотипных ветвящихся процессов. - Дискретн. матем., 1997, т. 9, № 1, с. 3-11.

89. Асимптотическое поведение вероятностей вырождения остановленных ветвящихся процессов. - Теория вероятн. и ее примен., 1998, т. 43, в. 2, с. 390-397.

90. О связи структурных характеристик прообразов и образов конечных множеств при некоторых классах отображений. - Труды по дискретной математике, 1998 , т. 2, с. $296-304$.

91. Ветвящиеся процессы: взгляд в прошлое. - Теория вероятн. и ее примен., 1999, т. 44, в. 3, с. 692-694.

92. Распределение вероятностей высоты генеалогического дерева многотипного ветвящегося процесса Гальтона-Ватсона. - Дискретн. матем., 1999, т. 11, № 1, c. 3-7.

93. Распределение вероятностей перманентов случайных матриц с независимыми элементами в поле $\mathrm{GF}(p)$. - Труды по дискретной математике, 2000, т. 3, c. $235-248$.

94. Минимальные точки надкритического ветвящегося блуждания на решетке $N_{0}^{r}$ и многотипные ветвящиеся процессы Гальтона-Ватсона. - Дискретн. матем., 2000, т. 12, № 1, с. 3-6.

95. Времена пребывания в конечном множестве состояний марковских ветвящихся процессов и вероятности вырождения одной модификации процесса ГальтонаВатсона. - Дискретн. матем., 2000, т. 12, № 4, с. 39-45.

96. Некоторые задачи, связанные с булевыми функциями. - Труды по дискретной математике, 2001, т. 4, с. 223-230.

97. Исследование вероятностной зависимости выхода автомата от некоторых характеристик входа. - Труды по дискретной математике, 2002, т. 5, с. 219-226.

98. Структурные характеристики некоторых неравномерных случайных отображений конечных множеств. - Труды по дискретной математике, 2002, т. 6, с. 184193.

99. Финальные вероятности для модифицированных ветвящихся процессов. - Дискретн. матем., 2002, т. 14, № 1, с. 3-10.

100. Ученый и воспитатель. - Вестник ИКСИ. Спец. выпуск, посвященный $100-$ летию академика А. Н. Колмогорова, 2003, с. 26-30.

101. Некоторые классы случайных отображений конечных множеств и неоднородные ветвящиеся процессы. - Дискретн. матем., 2004, т. 16, № 1, с. 9-13.

102. Курс теории вероятностей и математической статистики. 2-е изд. МоскваИжевск: ИКИ, 2004, 272 с.

103. Сходимость по распределению случайных отображений конечных множеств к ветвящимся процессам. - Дискретн. матем., 2005, т. 17, № 1, с. 18-21.

104. Асимптотика вероятности продолжения ограниченного снизу марковского критического ветвящегося процесса с непрерывным временем и бесконечной дисперсией. - Дискретн. матем., 2006, т. 18, № 1, с. 3-8.

105. Колмогоров в моей жизни и памяти. - В кн.: Колмогоров в воспоминаниях учеников. Редактор-составитель А. Н. Ширяев. М.: Изд-во МЦНМО, 2006, с. 196204.

106. Один класс докритических ветвящихся процессов с иммиграцией и с бесконечным числом типов частиц. - Дискретн. матем., 2007, т. 19, № 1, с. 6-10.

107. Сборник задач по теории вероятностей. 3-е изд., стер. Санкт-Петербург-Москва-Краснодар: Лань, 2009, 320 с. (совм. с В. П. Чистяковым, А. М. Зубковым).

108. Вычисление предельных вероятностей распределения перманента случайной матрицы в поле GF $(p)$. — Дискретн. матем., 2010, т. 22, № 3, с. 3-7. 